\title{
Comparative Rates of Adverse Events with 2-Hour versus 4-Hour Infusion of Total Dose Intravenous Iron Polymaltose
}

\author{
Arumugam Manoharan ${ }^{1,2,3^{*}}$, William Alexander ${ }^{2}$, Rajeev Ramakrishna ${ }^{1,2,3}$, Janet Legge ${ }^{2}$, John Uebel ${ }^{2}$ \\ ${ }^{1}$ Southern Sydney Haematology, Kogarah, Australia; ${ }^{2}$ Illawarra Private Cancer Care Centre, Wollongong, Australia; ${ }^{3}$ Graduate \\ School of Medicine, University of Wollongong, New South Wales, Australia. \\ Email: *Prof_Manoharan@uow.edu.au
}

Received December $20^{\text {th }}, 2013$; revised January $18^{\text {th }}, 2014$; accepted February $5^{\text {th }}, 2014$

Copyright (c) 2014 Arumugam Manoharan et al. This is an open access article distributed under the Creative Commons Attribution License, which permits unrestricted use, distribution, and reproduction in any medium, provided the original work is properly cited. In accordance of the Creative Commons Attribution License all Copyrights (C) 2014 are reserved for SCIRP and the owner of the intellectual property Arumugam Manoharan et al. All Copyright (C) 2014 are guarded by law and by SCIRP as a guardian.

\section{ABSTRACT}

Background and aim of the study: Patients with iron deficiency (ID) who are unable to tolerate or show sub-optimal response to oral iron therapy are candidates for parenteral iron therapy. This study evaluated the patient safety and tolerance of iron polymaltose given either as a 2-hour infusion or as a 4-hour infusion. Methods: A total of 243 patients with ID were randomized alternatively to receive iron polymaltose infusion either as a 2-hour infusion or as a 4-hour infusion. All patients received pre-medication with certizine hydrochloride 10 mg PO one-hour before infusion and hydrocortisone $50 \mathrm{mg}$ IVI immediately before infusion. Infusion related adverse events/side-effects during the infusion and over the next seven days were documented and graded as mild, moderate or severe. Results: The age of the patients ranged from 17 years to 92 years (mean 55.5 yr); M:F = 1:3.5. One hundred and twenty-two patients were in the 2-hour arm $(M: F=1: 5$; mean age $52.9 \mathrm{yr})$ and 121 were in the 4-hour arm (M:F = 1:2.6; mean age $58.1 \mathrm{yr})$. Iron infusion therapy was generally well tolerated by patients in both arms. Adverse events/side effects were documented in 14 patients in each arm (22 events in the 2-hour arm and 20 in the 4-hour arm) and included aches and pains, cannula-site pain/swelling, nausea, abdominal cramps, rash, vagal response, metallic taste, hot flushes and headaches. In most patients, these events were mild and none had any severe events. Conclusion: The incidence of adverse events with 2-hour infusion of iron polymaltose is similar to that observed with 4 -hour infusion of iron polymaltose.

\section{KEYWORDS}

Iron Deficiency; Rapid Iron Polymaltose Infusion

\section{Introduction}

Iron deficiency (ID) state, with or without anaemia, is a common problem worldwide afflicting all age groups for varying reasons [1]. Optimum management of patients with ID requires identification and treatment of the cause, as well as iron supplementation therapy, the latter generally given orally. However, there are several practical problems with oral iron therapy, e.g. gastro-intestinal side effects, poor absorption and poor patient compliance [2]. Patients with one or more of these problems are candidates for consideration of parenteral iron therapy. Sev-

${ }^{*}$ Corresponding author. eral recent reports have documented the efficacy and safety of total dose intravenous iron infusion therapy, making it the preferred parenteral route [3-5]. In Australia iron polymaltose is the only agent currently PBS (pharmaceutical benefit scheme) listed, given mostly as a 4-hour infusion [5,6]. In the present study, we have compared the safety and patient tolerance of 2-hour versus 4-hour infusion of total dose iron polymaltose in 243 patients.

\section{Material and Methods}

During a six month period (April-September, 2012) 243 
patients with proven ID and deemed unsuitable for oral iron therapy were randomized to receive total dose (500 $\mathrm{mg}-1000 \mathrm{mg}$ ) iron polymaltose intravenous therapy either as a 2-hour infusion or as a 4-hour infusion. The patients had been referred to one of the Consultant Clinical Haematologists for assessment and consideration of parenteral iron supplementation therapy. The rationale for the study was discussed with each patient by the Consultant Clinical Haematologist and the patients who had agreed to participate were randomized alternatively to the 2-hour arm or the 4-hour arm on the day of treatment by a reception staff member of the treating day care centre. Patients with the following medical history/problems were excluded from the study: hypersensitivity to iron, first trimester of pregnancy, severe inflammatory bowel disease, severe renal or liver disease, heart failure, bronchial asthma, history of severe allergic reactions and impending or recent surgery. The study was approved by the Human Resources Ethics Committee, University of Wollongong (HE 12/88) and all the participating patients had signed the required consent form.

Patients were routinely given pre-medication with certizine hydrochloride $10 \mathrm{mg} \mathrm{PO}$ one hour before the infusion and hydrocortisone $50 \mathrm{mg}$ IVI immediately before commencement of the infusion. Iron polymaltose (500 $\mathrm{mg}-1000 \mathrm{mg}$ ) was diluted in $500 \mathrm{ml}$ of normal saline and the infusion was commenced at $50 \mathrm{mls}$ per hour for 10 minutes in all patients. After ensuring that there are no anaphylactic reactions, the rate of infusion was increased to $125 \mathrm{mls}$ per hour for patients in the 4-hour group; and, to $250 \mathrm{mls}$ per hour for patients in the 2-hour group. They were closely monitored with periodic pulse and blood pressure measurements and for hypersensitivity reactions such as urticarial rash, itching, nausea and fever or shivering.

The nursing staff in the treating day care centre closely monitored the patients during the infusion and documented any adverse events. Subsequently, the patients were contacted one and seven days after the infusion to obtain information of possible delayed reactions: fever, arthralgia, myalgia, light headedness, headache, metallic taste, nausea, vomiting, diarrhoea and rash. The adverse events during and after the infusion were graded as mild (no observable patient discomfort/no activity limitation), moderate (observable patient discomfort/some activity limitation) or severe (distress or requiring medical assistance) [7].

\section{Results}

A total of 243 patients were recruited over a six month period from two centres (Sydney and Wollongong). The age of the patients ranged from 17 years to 92 years (mean $55.5 \mathrm{yr}$ ); $\mathrm{M}: \mathrm{F}=1: 3.5$. One hundred and twenty- two patients (M:F = 1:5; mean age 52.9 yr) received the iron infusion over a 2-hour period, whilst the other 121 patients $(\mathrm{M}: \mathrm{F}=1: 2.6$; mean age $58.1 \mathrm{yr})$ received the infusion over a 4-hour period. Iron infusion therapy was generally well tolerated and the majority of patients (in both arms) who returned to the clinical haematologists for follow-up reported an improved sense of wellbeing 1 7 days after the infusion.

The reported side-effects/adverse events in the 2-hour infusion arm and the 4-hour infusion arm are shown in Table 1. Fourteen of the 122 patients in the 2-hour infusion arm reported 22 side-effects/adverse events. Some of the side-effects (aches and pains, cannula-site pain/ swelling, nausea and abdominal cramps) lasted for more than 24 hours, whilst the others (rash, vagal response and metallic taste) were transient, lasting for less than one hour. In 12 of the 14 patients, the side-effects were mild; two patients had moderate aches and pains. None had any severe side-effects.

Table 1. Reports of adverse events.

\begin{tabular}{|c|c|c|c|c|c|c|}
\hline & \multicolumn{3}{|c|}{$\begin{array}{l}\text { 2-Hour infusion: } \\
\text { 14/122 patients }\end{array}$} & \multicolumn{3}{|c|}{$\begin{array}{l}\text { 4-Hour infusion: } \\
\text { 14/121 patients }\end{array}$} \\
\hline & Mild & Moderate & Severe & Mild & Moderate & Severe \\
\hline All & 20 & 2 & 0 & 17 & 3 & 0 \\
\hline \multicolumn{7}{|l|}{$\begin{array}{c}\text { During } \\
\text { infusion }\end{array}$} \\
\hline $\begin{array}{l}\text { Aches and } \\
\text { pains }\end{array}$ & & & & 2 & 0 & 0 \\
\hline Hot flushes & & & & 1 & 0 & 0 \\
\hline Rash & 1 & 0 & 0 & & & \\
\hline $\begin{array}{l}\text { Vagal } \\
\text { response }\end{array}$ & 1 & 0 & 0 & 1 & 0 & 0 \\
\hline \multicolumn{7}{|l|}{$\begin{array}{c}24 \text { Hrs } \\
\text { post-infusion }\end{array}$} \\
\hline $\begin{array}{l}\text { Abdominal } \\
\text { cramps }\end{array}$ & & & & 1 & 0 & 0 \\
\hline $\begin{array}{l}\text { Aches and } \\
\text { pains }\end{array}$ & 5 & 1 & 0 & 2 & 0 & 0 \\
\hline $\begin{array}{l}\text { Cannula site } \\
\text { pain/swelling }\end{array}$ & 1 & 0 & 0 & 1 & 1 & 0 \\
\hline Headache & & & & 2 & 0 & 0 \\
\hline Metallic taste & 2 & 0 & 0 & & & \\
\hline Nausea & 2 & 0 & 0 & & & \\
\hline \multicolumn{7}{|l|}{$\begin{array}{c}\text { Day 1-7 } \\
\text { post-infusion }\end{array}$} \\
\hline $\begin{array}{l}\text { Abdominal } \\
\text { cramps }\end{array}$ & 1 & 0 & 0 & & & \\
\hline $\begin{array}{l}\text { Aches and } \\
\text { pains }\end{array}$ & 5 & 1 & 0 & 4 & 1 & 0 \\
\hline $\begin{array}{l}\text { Cannula site } \\
\text { pain/swelling }\end{array}$ & 1 & 0 & 0 & 1 & 1 & 0 \\
\hline Headache & & & & 2 & 0 & 0 \\
\hline Nausea & 1 & 0 & 0 & & & \\
\hline
\end{tabular}


Fourteen of the 121 patients in the 4-hour infusion arm reported 20 side effects (Table 1 ). None of the side-effects was severe. Hot flushes, vagal response and abdominal cramps were mild and transient. Aches and pains lasted for less than 24 hours in two patients. In five patients this symptom lasted for more than 24 hours; in one of these patients this symptom was graded as "moderate". Two patients had cannula site pain and swelling which lasted for more than 24 hours; one "mild" and one "moderate". Two patients reported headache lasting for more than 24 hours—one "mild", one "moderate".

\section{Discussion}

Iron polymaltose therapy was well tolerated and the overall incidence of adverse events/side effects was low. There was no significant difference between the 2-hour infusion group and the 4-hour infusion group. In most of the patients (12/14, 11/14, respectively) the side-effects were mild; none had any severe side-effects. As the study was specifically designed to assess and compare the safety and patient tolerance and, a large proportion of the patients who participated in the study had returned to their regular medical practitioners for on-going care, data relating to the efficacy of iron polymaltose infusion were not routinely collected.

Our experience with the 2-hour infusion of iron polymaltose is comparable to that reported by Garg et al. [7] The latter authors administered iron polymaltose as a 58-minute infusion after an initial 15-minute test dose without pre-medication to 100 patients and documented 34 side-effects in 24 patients during infusion and 31 sideeffects in 26 patients during the 5-day post-infusion period. The presence of inflammatory bowel disease was associated with a higher incidence of side-effects during the infusion. None of the patients in our study had severe inflammatory bowel disease and they were routinely given pre-medication with cetirizine hydrochloride orally (one hour before infusion) and hydrocortisone IVI (immediately before infusion). These two differences may explain the lower incidence of side-effects documented in our study. However, we note that the potential beneficial role of pre-medication is controversial. A retrospective study of 386 patients by Newnham et al. [8] had shown no difference in the incidence of adverse events in patients receiving premedication (92 patients) compared to those (294 patients) who did not receive pre-medication.

Iron carboxymaltose (Ferric carboxymaltose) is another iron preparation which can be used for intravenous infusion therapy. This product can be given as a highdose (500 mg - $1000 \mathrm{mg}$ ) drip infusion over a 15 minute period. Although currently available in Australia, this product is not in the Pharmaceutical Benefit Scheme
(PBS) list and so, is more expensive ( $\$ 176.95$ for $500 \mathrm{mg}$ ) than iron polymaltose ( $\$ 50.36$ for $500 \mathrm{mg}$ ) [9]. The reported side-effects with iron carboxymaltose infusion are similar to those documented with iron polymaltose infusion: headaches, nausea, rash, dizziness, local injection site reactions, abdominal pain, diarrhoea and vomiting [10].

To the best of our knowledge, there are no reported studies comparing the rate of adverse events/side-effects between iron polymaltose infusion and iron carboxymaltose infusion. There are also no randomized studies to assess the benefits of pre-medication in patients receiving total dose iron infusion therapy. These reservations notwithstanding, the cumulative experience to date, has led to widespread and increasing use of total-dose iron infusion therapy as an effective, convenient and well-tolerated alternative for patients with ID who are deemed to be in need of parenteral iron therapy [11,12]. Infusions over shorter periods will enable Day Care Centres to treat more patients, thus reducing the patient waiting time and also making the treatment more cost-effective.

\section{REFERENCES}

[1] E. Beutler, "Disorders of Iron Metabolism in Williams Hematology,” In: M. A. Lichtman, E. Beutler, T. J. Kipps, U. Seligsohn, K. Kaushansky and J. F. Prchol, Eds., Williams Hematology, Mc-Graw-Hill, New York, 2006, p. 518.

[2] M. B. Zimmermann and R. F. Hurrell, "Nutritional Iron Deficiency,” Lancet, Vol. 370, No. 9586, 2007, pp. 511520.

[3] A. Daniilidis, A. Giannoulis and C. Pantelis, et al., "Total Infusion of Low Molecular Weight Iron-Dextran for Treating Post-Partum Anemia," Clinical and Experimental Obstetrics and Gynecology, Vol. 38, No. 2, 2011, pp. 159-161.

[4] F. Beigel, B. Lohn, R. P. Laubender, et al., "Iron Status and Analysis of Efficacy and Safety of Ferric Carboxymaltose Treatment in Patients with Inflammatory Bowel Disease,” Digestion, Vol. 85, 2012, pp. 47-54. http://dx.doi.org/10.1159/000333091

[5] M. L. Haines and P. R. Gibson, "Delayed Reactions to Total Dose Intravenous Iron Polymaltose,” Internal Medicine Journal, Vol. 39, No. 4, 2009, pp. 252-255. http://dx.doi.org/10.1111/j.1445-5994.2009.01898.x

[6] A. Manoharan, R. Ramakrishna and B. Pereira, "Delayed Adverse Reactions to Total-Dose Intravenous Iron Polymaltose," Internal Medicine Journal, Vol. 39, No. 12, 2009, p. 857. http://dx.doi.org/10.1111/j.1445-5994.2009.02077.x

[7] M. Garg, G. Morrison, A. Friedman, A. Lau, D. Lau and P. R. Gibson, "A Rapid Infusion Protocol Is Safe for Total Dose Iron Polymaltose: Time for Change,” Internal Medicine Journal, Vol. 41, No. 7, 2011 pp. 548-554. http://dx.doi.org/10.1111/j.1445-5994.2010.02356.x

[8] E. Newnham, I. Ahmad, A. Thornton and P. R. Gibson, 
"Safety of Iron Polymaltose Given as a Total Dose Iron Infusion,” Internal Medicine Journal, Vol. 36, No. 10, 2006, pp. 672-674.

http://dx.doi.org/10.1111/j.1445-5994.2006.01156.x

[9] Department of Health, "Guiding Principles for the Development of Intravenous (IV) Iron Infusion Practice,” Department of Health, Victoria, 2013.

[10] "Product Information: Ferinject (Ferric Carboxymaltose)
Vifor Pharma/Aspen Australia,” 2012.

[11] M. Naim and J. Hunter, "Intravenous Iron Replacement Management in General Practice," Australian Family Physician, Vol. 39, No. 11, 2010, pp. 839-841.

[12] A. K. Alhossain and A. E. Dennis, "Iron Deficiency Anaemia in Pregnancy and Postpartum: Pathophysiology and Use of Oral versus Intravenous Iron Therapy,” Journal of Pregnancy, 2012, Article ID: 630519. 